\title{
GROUP CHOICE AND EXTREMAL PROBLEMS IN THE ANALYSIS OP QUALITATIVE ATTRIBUTES
}

\author{
B.G. Mirkin \\ Inetitute of Economics \& Industrial \\ Engineering \\ Siberian Department of the USSR \\ Academy of Sciences \\ Novosibirsk, USSR
}

A problem of aggregating incoherent "individual" data on a fixed set of objects for obtaining their common description has gained recently an increasing urgency. In one case they can be individual opinions of jury members about preference order conceming objects considered which are to be reduced to a common "group" decision. In another, they may be inconsistent indicatorg of objects quality which are to be reduced to a single compromise indicator; in still another, they are different classifications of objects chosen as initial ones in building a reconciled classification.

According to the established notions of the group choice theory $[1]-[3]$ we shall refer to this problem as a group choice problem, and to the rule of transition to a "group" set of data, as a reconciling principle.

We shall assume that the data are given non-quantitatively, i.e. they are described by binary relations on a given set of objects A. If $R \subseteq A \times A$ describes a preference relation, then the fact of belonging $(x, y) \in R$ denotes that $x$ is not less preferable than $y$ - If $R$ corresponds to some quality indicator, $(x, y) \in R$ denotes that $x$ is not worse than $y$ by this indicator. If $R$ is defined by some classification of objects, then $(x, y) \in R$ denotes the fact of $x$ and $y$ belonging to the same class. To different types of data, of course, different types of binary relations correspond. Ordinarily, examined are nominal attributes, set by equivalence 
relations and rank ones, set by complete preorders (rankings).

In ref. [4] four requirements to reconciling principles are described (monotony, universality, independence of objects, sovereignty of group choice) similar to the appropriate axioms of Arrow, and it was shown that these requirements entirely guarantee the so called reconciling principles. The reconciling principle $F\left(R_{1}, \ldots, R_{n}\right)$ is called trivial if there exists such a subset $V$ of the set of indices $\{1,2, \ldots, n\}$ that $F$ coincides with $F_{V}$ defined by the formula:

$$
F_{V}\left(R_{1}, \ldots, R_{n}\right)=\bigcap_{\kappa \in V} R_{K} \text {. }
$$

Trivial principles are broadly used in reconciling classifications. Now we shall consider a more complicated reconciling principle, i.e. a rule of majority which does not satisfy the axiom of universality [4].

The rule of majority allows different interpretations. We shall denote by $a_{x y}$ the number of such relations in the set $R_{1}, \ldots$ $\ldots, R_{n}$ which contain the pair of objects $(x, y)$. According to one interpretation, the group relation $R$ is defined by the relation

$$
(x, y) \in R-a_{x y} \geqslant a_{y x}
$$

and according to another, by relation

$$
(x, y) \in R \longrightarrow a_{x y} \geqslant \frac{n}{2}
$$

The difference between the rules (1) and (2) can be explained by the following example. Suppose 100 persons have expressed their opinions about comparative preference concerning two objects $x$ and $y$. For 99 persons the objects turned out to be similar by preference, and for one person $x$ was better than $y$. This means that 99 individual relations contain both $(x, y)$ and $(y, x)$, and one relation contains only $(x, y)$ so that $a_{x y}=100$, $a_{y x}=99$. Then, according to the rule (1), $x$ is better than $y$, while $x$ and $y$ are similar by preference in the sense of (2). Roughly speaking, the rule (1) expresses the opinion of the "active minority", while the rule (2), the opinion of the "passive majority". If, however, only strict preferences are congidered, it is easy to see that both rules yield the same results.

We shall examine the following natural modification of the rule (2). The relation $R$ will be called majoritary if it satisfies the condition (2) for all pairs of objects $(x, y)$ with possible 
exceptions of such pairs $(x, y)$ for which $a_{x y}=\frac{n}{2}$.

The set of relations $D$ will be called permissible if for any set of relations $R, \ldots, R_{n}$ from $D$ (not necessarily different) there exists a transitive majoritary relation. The set of three strict rankings of type $(x, y, z),(y, z, x),(z, x, y)$ derived from each other through cyclic permutation of objects will be referred to as cyclic. Each of the cyclic set orderings generates the remaining ones through the permutation of the first object to the last place and of the last object to the first place; these two objects will be called cyclic. A set of three non-strict rankings $\{x Q y Q z, y Q z Q x$, $z Q x Q y\}$ (here symbol $x Q y$ denotes a relation: $x$ is preferable or indifferent to $y$ ) will be called cyclic if it satisfies the following conditions:

a) if in one of these rankings all three objects $x, y, z$ are indifferent between themselves, then for the cyclic objects of the remaining rankings a strict preference occurs;

b) all three rankings cannot be uniformly dichotomic, i.e. they must not partition the objects $x, y$ and $z$ into two classes so that a one-element class in each ranking precedes a two-element one (or in each ranking follows the two-element one).

Theorem 1. The set of rankings $D$ is permissible if and only if it does not involve a cyclic set for any three objects.

Now we shall consider the natural extension of the majority rule (2) in terms of distance between the relations, applicable also to inadmissible sets of relations. For two relations $R$ and $P$ for distance $d(P, R)$ the number of pairs in their symmetric difference

$$
d(P, R)=|P \Delta R|=|(P-R) U(R-P)|
$$

will be taken.

Let $R_{1}, \ldots, R_{n}$ be given relations from $D$. The relation $R^{*} \in E$ minimizing the sum of distances $f(R)=$ $=\sum_{K=1}^{n} d\left(R, R_{K}\right)$ on all $R \in E \quad$ will be referred to as a median of system $R_{1}, \ldots, R_{n}$ in class $E$. As a reconciling principle the procedure of taking the median in class $E$ will be considered.

If rankings are only examined, then the distance between the appropriate relations is proportional to the distance axiomatically inserted in $/ 5]$ (see $/ 6 /$ ). 
It is easy to show that the procedure of taking the median indeed generalizes the majority rule: if majoritary relation belongs to the class $E$, it is the median in the class $E$.

The procedure of taking the median can be stated in terms of variables $a_{x y}$ characterizing the number of relations $R_{1}, \ldots, R_{n}$ containing the pair $(x, y)$. Thus the following statement takes place.

Theorem 2. The relation $R^{*}$ is the median of the system $R_{1}, \ldots, R_{n}$ in class $E$ if and only if it maximizes the function

$$
g(R)=\sum_{(x, y) \in R}\left(a_{x y}-\frac{n}{2}\right), \quad R \in E .
$$

In particular, if $E$ consists only of relations corresponding to strict rankings, so that any $R \in E$ is marked by strict ranking of objects $\left(i_{1}, \ldots, i_{n}\right) \quad(N$ the number of objects), i.e.

$$
\begin{aligned}
& R=\left\{\left(i_{K}, i_{l}\right) \mid K \leqslant l, K, Z=1, \ldots, N\right\} \text { the function } g \text { is } \\
& g(R)=g\left(i_{1}, \ldots, i_{N}\right)=\sum_{K<l} a_{i_{K}} i_{l}+\text { const. }
\end{aligned}
$$

In this case, the maximization problem (3) is equivalent to the problem of such simultaneous permutation of colums and lines in the matrix $\left\|\alpha_{i j}\right\|_{1}^{N}$ which would maximize the sum of overdiagonal elements $\sum_{k<l} a_{i_{k}} i_{l}$. The algorithms for this problem are given in

In search for the median in a class of equivalence relations corresponding to certain partitions of a set of objects (in solving the problem of reconciling different classifications or nominal attributes [9-107) the problem (3) is concretized into a problem of constructing such a partition $R=\left\{R^{1}, R^{2}, \ldots, R^{m}\right\}$ of the set of objects into non-overlapping classes $R^{S}$ which would maximize the function

$$
g(R)=\sum_{s=1}^{m} \sum_{i, j \in R}\left(a_{i j j}-\frac{n}{2}\right) .
$$

The problem (4) is quite analogous to the known "intuitive" statements of the problem of automatic classification of a set of interconnected objects, and as indicators of interconnection, the vaxiables $a_{i j}-\frac{n}{2}$ serve here.

The median in the context of data analysis is interpreted as "intermal" factor representing "extemal" attributes $R, \ldots, R_{n}$. 
Of interest is a search for intermal factor in a class of relations characterizing the partitions $R=\left\{R^{\prime}, \ldots, R^{m}\right\}$ of the set of objects with a structure of "essential" connections between the classes set by the relation $\mathscr{C} \subseteq\{1,2, \ldots, m\} \times\{1, \ldots, m\}$. The median in a class of these relations is a solution to a problem of maximization of the following function:

$$
g(R, x)=\sum_{(s, t) \in \mathscr{X}} \sum_{i \in R} \sum_{j \in R^{t}}\left(a_{i j}-\frac{n}{2}\right) .
$$

The optimal partition $R$ with a "structure" $\mathscr{C}$ characterizes the aggregated representation of "essential" connections in the matrix $\left\|a_{i j}\right\|_{1}^{N}$ which is, generally speaking, different from the unordered partition obtained within automatic classification.

In collaboration with V.I.Kupershtokh and V.A.Trofimov an algorithm has been developed for building "practically" optimal $R$ and $\partial$ which was applied to the analysis of real association matrices in economics and biology.

We shall describe only two examples:

1. The analysis of the stmucture of intershop deliveries (in collaboration with G.V. Grenback).

Considered was a matrix $a_{i j}$ where $i, j$ are numbers of shops on a Novosibirsk plant, and $a_{i j}$ the quantity of titles of parts going from the $i-t h$ shop to the $j-t h$ shop. In this example from $\alpha_{i j}$ we subtracted the arithmetic mean $\bar{a}=[1 / N(N-1)] \sum_{i \neq j} a_{i j}$ instead of $\frac{n}{2}$. The obtained atructure basically coincided with the existing structure of the plant, and existing deviations made it possible to invite the management of the plant to introduce certain changes into the organization structure which aimed et the relaxation of tensions for the management.

2. The analyais of genetic structures (in collaboration with S.H.Rodin).

As objects, there were considered genetic mutations $i, j=1, \ldots, 36$ and $a_{i j}=1$ if the interbreeding of mutants $i$ and $j$ does not lead to descendants, and $a_{i j}=0$ otherwise.

The structure analysis has supported the genetic hypothesis that mutations affecting the same functional centers (sites) of albumen must in the same way interact with other mutations. Apart from this, it became possible to reveal the number of sites and the structure of their interaction. 
1. Luce, R., H.Raiffa

Games and Decisions.

Wiley, N.Y. 1957.

2. Arrow, K.J.

Social Choice and Individual Values.

Wiley. N.Y. 1951.

3. Sen, A.K.

Collective Choice and Social Welfare.

Oliver and Boyd, 1970.

4. Mirkin, B.G.

On Principles of Reconciling Relations. (Russian)

Kibermetika. Kiev. 1973, no.2.

5. Kemeny, J., J. Snell. Mathematical Models in the Social Sciences.

Elsevier. N.Y. 1963.

6. Mirkin, B.G., L.B.Chorny

Some Properties of Space of Partitions.(Russian)

Mat. Analiz Ekon. Model., part III, Institute of Economica of the Siberian Dept. of the USSR AS. Novosibirsk. 1972.

7. Burkov, V.N., V.O.Groppen

Cuts In Heavily Associated Graphs and Permutation Potentials (Rusgjan). Avt. I telem. 1972, no.6.

8. Iitrak, B.G. On Ordering Objects by Preferences (Russian).

Mat. met. upr. proizv., issue 5, the University of Moscow, Hoscow, 1973.

9. Mirkin, B.G.

Approximation Problems in the Space of Relations and

the Analysis of Qualitative Attributes (Russian).

10. Mirkin, B.G. Avt. i telem. 1974, no.9.

On an Approach to the Analysis of Primary Sociological Data.

Paper submitted at the VII World Congress of Sociology, Institute of Economics of the Siberian Dept. of the USSR AS. Novosibirak. 1970. 